\title{
Evaluation on Finger-Vein Identification Process
}

\author{
Shwetambari Kharabe, C.Nalini
}

\begin{abstract}
The finger vein demand system plays a vivacious development for individual ID and check. In constant time, this system is getting approval, as it gives a high security and comfort approach for individual authentication. In this paper we concentrated on the structure study on different advances which are checked with the game-plan of finger vein seeing affirmation and verification. Clarified steps are finger Vein Data checking, finger Veinpre-sorting out, finger Veinsegmentation, finger Veinfeature extraction and finger Vein outline and managing. In setting on the assessment, the perfect philosophy and system is seen, which will be a calming answer for finger vein endorsing.
\end{abstract}

\section{INTRODUCTION}

Analysis and review being done on various techniques applied for finger vein recognition system by eminent personalities. A brief analysis of each method was carried out to identify its own merits and limitation. The literature survey serves as stare of the art tool to develop an efficient method which can be used as authentication tool based on finger vein. The survey helps to gather novel ideas from the existing methods such as capturing of finer image without noise, enhancing the image captured segmentation technique and matching process.

\section{RELATED WORK}

\section{a) Literature Survey on Finger Vein Data achievement}

During the time spent picture checking, vein pictures are found using infrared scanner. Gotten pictures effect beat condition conditions ought precision. Picture affirming sort for instance segregated and online $[2,3]$.

David Mulyonoet. Proposes an ideology for finger vein figures for off-line and a online acquisition. The figures which is acquired during the course of real time is called On-line images. Images which are acquired from already existing source such as database, historical information, etc are called as Off-line images. By using equipment like Web Camera or any device so designed using light transmission technology are the distinct methods for capturing on-line images. Light reflection and transmission are the two prominent methods used for acquiring on-line images and the prominent difference between the two is the location where in the near infrared light is positioned. As a result of light reflection methodology, the instance of finger vein is obtained dependent on close infrared light reflection from palmer surface at any rate in the system for light transmission the relative is set model is gotten.

Revised Manuscript Received on August 14, 2019.

Shwetambari Kharabe, Phd Scholar, Department of CSE, Bharath Institute of Higher Education and Research, Chennai, Tamilnadu, India. (Email: srkharabe16@gmail.com)

C.Nalini, Professor, Department of CSE, Bharath Institute of Higher Education and Research, Chennai,Tamilnadu, India.(Email: drnalinichidambaram@gmail.com)
Notwithstanding the way that in light transmission system, the which enters to catch finger vein plan. While comparing between light reflection and transmission methods, the later one can acquire vein images with high contrast, and most of the devices apply this methodology[6,7].

\section{b) Literature Survey on Finger Vein Pre-processing}

In finger vein based biometric systems, various preprocessing task are to be performed - retrieving information regarding edges, enhancement of contrast and brightness, noise removal, sharpening of images, etc. captured. These pre-processes helps to improve image quality, which can be used as an input during later stage of process for obtaining more relevant information and authentication tool. Indeed, if the quality of the image is better, then better accuracy can be gained which helps to improve upon the authenticity of the biometric system. Primarily the pre-processing activity involves segmentation of finger vein images and alignment [8-11], denoising of images, detection of Region of Interest (ROI), normalization.

\section{c) Literature Survey on Finger Vein Segmentation}

In picture division the authentic picture is separated from its experience what's more mayhem is cleared up reasonably in any case it is amazingly hard to empty exact subtleties of the vein plan on account of sporadic commotion. Edge picture system of utilized registers makes edges each and makes twofold picture for example picture with just diminish or white disguising. The most central piece of this operation will be the affirmation of a solitary edge respect which is the summit estimation of the picture and called binarization[2,3].

Fernando C. Monteiro [12,13] proposed a novel system for fragmenting finger vein picture subordinate uneasy data which can be acquired by watershed of morphological check and creepy procedure. By executing pre-arranging experience of from the beginning reducing the commotion by utilizing correlative channel and therefore starter division is performed for district consolidating also region similarity. At long last by utilizing Multi-class Normalized Cut Method is incited for diagram based zone gathering. This system is estimation multifaceted nature notwithstanding the path that out performed over different procedures.R. V. Patil [14] prerogative that better result can be provided by K-means image segmentation, if the estimation of cluster numbers are measure accurately. The process of edge detection is considered as major phenomenon for estimating the cluster numbers accurately. To detect the edges and find cluster,

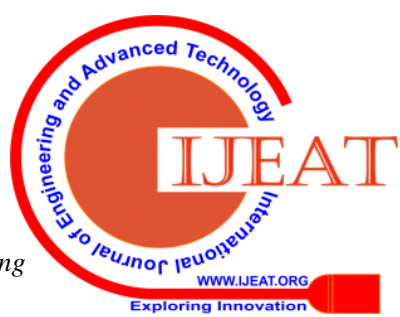


phase congruency was proposed by the author. The clusters are made and identified based on Threshold and Euclidean distance computation and the K-means. Results obtained based on experiments on nine different images proves that the identification of clusters based on the proposed method was accurate and optimal.

Feature extraction is a one of a kind sort of dimensionality decline that changes input data into the course of action of features. Collection of counts are used for this methodology moreover channels, for instance, center channel, Gabor channel, SIFT, points of interest, accurate measures, close-by parallel model, skeleton system are used for feature extraction to evacuate the features of vein without tumult and contorting. To develop significantly precise individual conspicuous verification systems, Fingervein pat-terns should be expelled unquestionably from the got pictures, and the method must be executed conveniently in order to satisfy requirements[2,3].

In order to think finger vein structures from non-uniform pictures, by a solid system, the procedure proposed should consider totrack diminish lines appears in an image more than once. In light of the quantum of times, wherein the accompanying lines experience the centers, the isolating strategy is portrayed. A few abstract works disseminated by popular characters, depicting the technique and modalities for isolating finger vein pictures are discussed in this work.

N. Miura, A. Nagasaka, and T. Miyatake $[1,15]$ proposed subject to repeated line following methodology. This strategy grasps the case of extraction by applying number of times the accompanying lines experience the points[15]. This technique recognizes close-by dull lines, which starts at various positions for line following and the proportionate is executed by moving nearby the lines by pixel by pixel.. During this method in case a dull line isn't noticeable, by then start another after action from another circumstance until all the diminish lines present in an image is recognized. This technique is accomplished more than once, by executing close-by line following assignments and subject to this exercises the region of the lines spread and the finger veins model are verifiably gotten. Regardless of the way that we can track noises similarly by applying this strategy, yet is underlined to a smaller degree than the diminish lines. This proposed procedure improves the quality of line extraction technique and to diminish the accompanying movement sum. This methodology also reduces the computational cost in regards to spatial decline of the model when stood out from other standard systems.

M..Subramani [20] offered another advancement for expelling plan from better vein pictures in idea with directional information of a comparative using Radon Transform and Principle Component Analysis (PCA) methodology [16]. The basic features of the vein pictures are evacuated by applying Radon projections different way for each projection. The estimation of picture control vector at a predefined edge is done by using this figuring reliant on decided purpose of organized radian line. The assignment cross section is created based count using this figuring, from the individual projection. In order to make a segment vector, the PCA examination is performed on the projection cross

\section{d) Literature Survey on Finger Vein withdrawal}

section for determining specific characteristics and arranging them in jumping demand. Therefore enrolled component vector will be valuable in describing the finger vein picture unquestionably.

e) Literature Survey on Finger Vein Image Classification and Matching organization

Fein Vein approach is doling out pixels in the picture to requesting or classes of premium subject to learning techniques and highlights. Sorts of learning technique are Supervised Learning and Unsupervised learning. In Supervised Learning technique is de-separate to shape a mapping from one heap of factors (information) to and-other strategy of parts (data classes) and review pulled in with the learning structure. The classifier has the upside of an inspector or zone information utilizing which the classifier can be guided to get settled with the association between the information and the classes. The extent of classes, model pixels for each class can be seen utilizing this earlier information.

Solo learning occurs without a screen and Exploration of the information space to find the reasonable laws basic the information improvement. Unequivocally when access to space information or the experience of a pro is feeling the loss of, the information can in any case be neediness stricken some spot close numerical assessment, whereby the information are amassed into subsets or packs subject to quantifiable similarity.

Regulated assembling by and large performs superior to anything autonomous depiction if uncommon quality preparing information is accessible solo classifiers is used to do key assessment of information before coordinated solicitation. In Features depiction system highlight are qualities of the information fragments subject to which the portions are distributed to different classes [2, 3].

\section{Arranging}

Orchestrating is one of the huge stage in finger vein evident assertion. After features are removed from the vein picture the orchestrating stage evaluates the comparability or separation between the data finger vein picture features and the beginning late picked ones in the database $[2,3]$.

(a)Solicitation and Matching system is one of the basic stage in finger vein prominent proof. This framework checks the similarity or distinction among the steady data finger vein pictures and recorded pictures starting late set away in the database. This is the methodology which is performed expediently once the finger vein highlights are extracted [17-19]. Exactly when this framework finished, by then the going with stage is minutia sorting out which fuses

\section{(a) False_Removing}

(b) Score Calculating template

Literature survey is carried on based on the papers published by various authors on their contribution towards these process are evaluated and submitted in this thesis work. 
Fei Liu et al proposed a new method for minutiae pairing which is called Singular Value Decomposition (SVD) [20]. The process of False Removing is performed based on Local Extensive Binary Pattern (LEBP) by applying the local characteristics which are rich in nature for minutiae representation. LEBP is the combination of Local Directional and Local Multilayer binary pattern. For image classification Support Vector Machine (SVM) is widely used for image classification and matching.

\section{RESULTS \& DISCUSSIONS:}

With the extensive analysis of literature works, we can envisage the horizon to overcome the limitations understood during the course of literature work, that the proposed methods should built upon. Based on the understanding, we proposed to develop a robust finger vein based identification system, which has assumption that the unique feature of vein pattern will be a milestone towards the personal identification system using biometrics.

The identification recently evolving biometric had been considerably focused of biometric based miniature size image capturing devices which is used to capture vein images helps to achieve miniaturizing the finger vein authentication technology.

\begin{tabular}{|l|l|l|}
\hline \multicolumn{1}{|c|}{ Components } & \multicolumn{1}{|c|}{ Author } & \multicolumn{1}{c|}{ Methodology } \\
\hline $\begin{array}{l}\text { Finger Vein Data } \\
\text { Acquisition }\end{array}$ & David Mulyono et. al & $\begin{array}{l}\text { Off-line and online } \\
\text { acquisition, Light reflection } \\
\text { and transmission are the two } \\
\text { prominent methods used }\end{array}$ \\
\hline Finger Vein Pre-processing & A. Gayathri, A Srinivasan & $\begin{array}{l}\text { Retrieving information } \\
\text { regarding edges, enhancement } \\
\text { of contrast and brightness, } \\
\text { noise removal, sharpening of } \\
\text { images }\end{array}$ \\
\hline Finger Vein Segmentation & Fernando C. Monteiro & $\begin{array}{l}\text { A novel method for } \\
\text { segmenting finger vein image } \\
\text { based on edge information } \\
\text { which can be obtained by } \\
\text { watershed of morphological } \\
\text { algorithmand spectral method }\end{array}$ \\
\hline Finger Vein Extraction & $\begin{array}{l}\text { N. Miura, A. Nagasaka, and } \\
\text { T. Mivatake }\end{array}$ & $\begin{array}{l}\text { A new method for feature } \\
\text { extraction based on repeated } \\
\text { line tracking technique }\end{array}$ \\
\hline $\begin{array}{l}\text { Finger Vein Image } \\
\text { Classification and Matching }\end{array}$ & Fei Liu et al & $\begin{array}{l}\text { A new method for minutiae } \\
\text { pairing which is called } \\
\text { SingularValue Decomposition }\end{array}$ \\
\hline
\end{tabular}

\section{CONCLUSION}

In light of this piece study, exhibits an appraisal of various systems utilized during the time spent finger-vein division, join extraction for biometric accreditation and seeing certification. This examination paper shows the frantic benchmarks, explicit division systems and highlight extraction procedures and execution assessment estimations saw all through finger vein clear affirmation. Investigating the unquestionable check standard, affiliations and business use encounters, unequivocal Finger-vein Feature Extraction Techniques offer various degrees of execution, trashing impediment, quality, security and precision. A careful assessment of need and structure execution will be useful for winding up new advantageous fuse with better execution in the appraisal work.For the future research work we want to consider, a strategy approach dependent on a guided programming program, which can utilize the twofold designs in the undeniable affirmation framework.

\section{REFERENCES}

1. N. Miura, A. Nagasaka and T. Miyatake, Extraction of finger-vein patterns using maximum curvature points in image profiles, IEICE Trans. Inf. \& Syst. Vol.90, no.D(8),pp. 1185-1194, 2007.

2. ShwetambariKharabe, C. Nalini," Survey on Finger-Vein Segmentation and Authentication", International Journal of Engineering \& Technology, 7(1.2) (2018), 9-14.

3. ShwetambariKharabe, C. Nalini," Robust ROI Localization Based Finger Vein Authentication Using Adaptive Thresholding Extraction with Deep Learning Technique", Jour of Adv Research in Dynamical \& Control Systems, Vol. 10, 07-Special Issue, 2018.

4. Xin Ma, Xiaojun Jing, Hai Huang, Yuanhao Cui, Junsheng Mu "Palm vein recognition scheme based on an adaptive Gabor filter", January 2017

5. Jun Wang, Guoqing Wang "Quality-explicit hand vein acknowledgment framework", January 2017 Edward Rosten, Reid Porter, and Tom Drummond "Quicker and Better: A Machine Learning Approach to Corner Detection",January 2010

6. Chih-Hsien Hsia, Jing-Ming Guo ,Chong-Sheng Wu "Finger-vein recognition based on parametric-oriented corrections",December 2016

7. D. Huang, R. Zhang, Y. Yin, Y. Wang, and Y. Wang, "Neighborhood highlight way to deal with dorsal hand vein acknowledgment by centroid-based round key-point matrix and fine-grained coordinating" , February 2017

8. A.Gayathri, A.Srinivasan, "An efficient algorithm for image denoising using NLM and DBUTM estimation", TENCON 2014- 2014 IEEE Region 10 Conference, pg.no 1-6, 2014.

9. A.Gayathri, A.Srinivasan, "Wavelet Based Self Adaptive Dictionary Algorithm for Image Denoising", Advances in Natural and Applied Science 9(9). 7-17, 2015.

10. A.Gayathri,"Photometrical and Geomentrical similar patch based image denoising using Wavelet Decomposition", Journal of Medical and Bioengineering 4, 47-53, 2015.

11. A.Gayathri and V.Nandhini, "HVS based Enhanced Medical Image Fusion",Computational Intelligence and Information Technology, 250, 870-872, 2011.

12. F. C. Monteiro and A. Campilho, "Watershed framework to region-based image segmentation," in Proc. International Conference on Pattern Recognition, ICPR 19th, pp. 1-4, 2008.

13. M. Hameed, M. Sharif, M. Raza, S. W. Haider, and M. Iqbal, "Framework for the comparison of classifiers for medical image segmentation with transform and moment based features," Research Journal of Recent Sciences, vol. 2277, p. 2502, 2012

14. R. Patil and K. Jondhale, "Edge based technique to estimate number of clusters in k-means colour image segmentation," in Proc. 3rd IEEE International Conference on Computer Science and Information Technology (ICCSIT), pp. 117-121, 2010.

15. N. Miura, A. Nagasaka, and T. Miyatake, "Feature extraction of finger vein patterns based on repeated line tracking and its application to personal identification", Machine Vision Application, vol. 15, no.4, pp.194-203, 2004.

16. M.Subramani, "Highly secure and reliable user identification based on finger vein patterns" Double Blind Peer Reviewed International Research Journal, 11, 2011.

17. Jian-Da Wu, Chiung-Tsiung Liu "Finger Vein Pattern 
Identification using SVM and Neural Network Technique" Elsevier, Expert Systems with Applications 38(2011) 14284-14289.

18. PandillapalliGowtham, Ch.sindhu, C.Sudheer Kumar, Ch.ChandraSekar "Identification of Finger Images using Score-Level Fusion" International Journal of Engineering Science Invention, Volume 2 Issue 2,February 2013.

19. Cheng-Bo Yu, Hua-Feng Qin, Lian Zhang, Yan-Zhe Cui "Finger Vein Image Recognition Combining Modified Hausdorff Distance with Minutiae Feature Matching" J.Biomedical Science and Engineering, 2009.

20. D. Mulyono and S.-J. Hong, "A study of finger-vein biometric for personal identification," in Proc. IEEE Int. Symp.BiometricsSecur. Technol., Apr. 2008, pp. 1-8.

21. E. C. Lee and K. R. Park, "Restoration method of skin scattering blurred vein image for finger-vein recognition," IET Electron. Lett., vol. 45, no. 21, pp. 1074-1076, 2009.

22. Dr.S.P. Anandaraj "Research Opportunities and Challenges of a Security Concerns associated with Big Data in Cloud Computing", IEEE International conference on ISMAC IoT in Social, Mobile, Analytics and Cloud published in IEEE Explore, DOI: 10.1109/ISMAC.2017.8058278, Electronic ISBN: 978-1-50903243-3,Print ISBN: 978-1-5090-3242-6,10th to 11th Feb, 2017

23. N. Miura, A. Nagasaka, and T. Miyatake, "Feature extraction of fingervein patterns based on repeated line tracking and its application to personal identification," Mach. Vis. Appl., vol. 15, no. 4, pp. 194-203, 2004.

24. J. Peng, N. Wang, A. A. A. El-Latif, Q. Li, and X. Niu, "Finger-vein verification using Gabor filter and SIFT feature matching," in Proc. IEEE Int. Conf. Intell. Inf. Hiding Multimedia Signal Process., Jul. 2012, pp. 45-48.

25. H. G. Kim, E. J. Lee, G. J. Yoon, S. D. Yang, E. C. Lee, and S. M. Yoon, "Illumination normalization for SIFT based finger vein authentication," in Proc. Int. Symp. Vis. Comput., 2012, pp. 21-30.

26. Kono, M.; Ueki, H.; Umemura, S. Near-infrared finger vein patterns for personal identification. Appl. Opt. 2002, 41, 7429-7436.

\section{AUTHORS PROFILE}

Shwetambari Kharabe, Phd Scholar, Department of CSE, Bharath Institute of Higher Education and Research, Chennai, Chennai, India.

Email: srkharabe16@gmail.com

C.Nalini, Professor, Department of CSE, Bharath Institute of Higher Education and Research, Chennai, Chennai, India.

Email: drnalinichidambaram@gmail.com 